\title{
Inter-Laboratory Ring Trial to Evaluate Reverse Transcription Polymerase Chain Reaction Methods Used for Dolphin Morbillivirus Detection in Italy
}

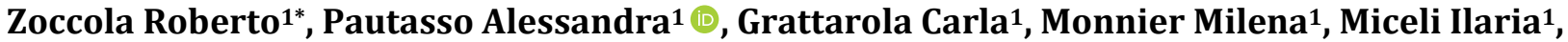 \\ Ingravalle Francesco ${ }^{1}$, Crescio Maria Ines ${ }^{1}$, Giorda Federica ${ }^{1}$, Masoero Loretta ${ }^{1}$, \\ Zaccaria Guendalina ${ }^{2}$, Purpari Giuseppa ${ }^{3}$, Cersini Antonella ${ }^{4}$, Viscardi Maurizio5, \\ Serracca Laura ${ }^{6}$, Padalino Iolanda ${ }^{7}$, Puggioni Giantonella ${ }^{8}$, Canonico Cristina ${ }^{9}$, Toffan Anna ${ }^{10}$, \\ Centelleghe Cinzia11, Di Francesco Cristina Esmeralda12, Mazzariol Sandro' ${ }^{11}$, \\ Di Guardo Giovanni ${ }^{12}$, Mignone Walter ${ }^{13}$, Casalone Cristina1 ${ }^{10}$, Goria Maria1
}

\footnotetext{
${ }^{1}$ Istituto Zooprofilattico Sperimentale del Piemonte Liguria e Valle d'Aosta, Torino, Italy

${ }^{2}$ Istituto Zooprofilattico Sperimentale dell'Abruzzo e del Molise, Teramo, Italy

${ }^{3}$ Istituto Zooprofilattico Sperimentale della Sicilia, Palermo, Italy

${ }^{4}$ Istituto Zooprofilattico Sperimentale del Lazio e della Toscana, Roma, Italy

${ }^{5}$ Istituto Zooprofilattico Sperimentale del Mezzogiorno, Portici (NA), Italy

${ }^{6}$ Istituto Zooprofilattico Sperimentale del Piemonte Liguria e Valle d'Aosta, La Spezia, Italy

${ }^{7}$ Istituto Zooprofilattico Sperimentale della Puglia e Basilicata, Foggia, Italy

${ }^{8}$ Istituto Zooprofilattico Sperimentale della Sardegna, Sassari, Italy

${ }^{9}$ Istituto Zooprofilattico Sperimentale dell' Umbria e delle Marche, Ancona, Italy

${ }^{10}$ Istituto Zooprofilattico Sperimentale delle Venezie, Legnaro (PD), Italy

${ }^{11}$ University of Padova, Department of Comparative Biomedicine and Food Science (BCA), Legnaro (PD), Italy

${ }^{12}$ Faculty of Veterinary Medicine, University of Teramo, Teramo, Italy

${ }^{13}$ Istituto Zooprofilattico Sperimentale del Piemonte Liguria e Valle d'Aosta, Imperia, Liguria, Italy

Email: *roberto.zoccola@izsto.it, Alessandra.pautasso@izsto.it, Carla.grattarola@izsto.it, milena.monnier@izsto.it,

ilaria.mic@gmail.com, francesco.ingravalle@izsto.it, MariaInes.crescio@izsto.it, federica.giorda@izsto.it, loretta.masoero@izsto.it, g.zaccaria@izs.it, giuseppa.purpari@izssicilia.it, antonella.cersini@izslt.it, maurizio.viscardi@cert.izsmportici.it,

laura.serracca@izsto.it, iolanda.padalino@izspb.it, giantonella.puggioni@izs-sardegna.it, c.canonico@izsum.it, atoffan@izsvenezie.it, cinzia.centelleghe@unipd.it, cedifrancesco@unite.it, sandro.mazzariol@unipd.it, gdiguardo@unite.it, walter.mignone@izsto.it,

cristina.casalone@izsto.it, maria.goria@izsto.it
}

How to cite this paper: Zoccola, R. , Pautasso, A. , Grattarola, C., Monnier, M. , Miceli, I., Ingravalle, F., Crescio, I., Giorda F., Masoero L., Zaccaria, G., Purpari, G., Cersini, A., Viscardi, M., Serracca, L., Padalino, I., Puggioni, G., Canonico, C., Toffan, A., Centelleghe, C., Di Francesco, C.E., Mazzariol, S., Di Guardo, G., Mignone, W., Casalone, C.

\begin{abstract}
Dolphin Morbillivirus (DMV) is one of the most frequently detected pathogens in stranded cetacean specimens worldwide as well as in Italy. Due to the persistence of DMV in the Mediterranean Sea and to the lack of information about the efficiency of the available diagnostic techniques, the Italian National Reference Centre for diagnostic activities on dead stranded marine mam-
\end{abstract}


and Goria, M. (2019) Inter-Laboratory Ring Trial to Evaluate Reverse Transcription Polymerase Chain Reaction Methods Used for Dolphin Morbillivirus Detection in Italy. Advances in Microbiology, 9, 423-437. https://doi.org/10.4236/aim.2019.95025

Received: March 18, 2019

Accepted: May 7, 2019

Published: May 10, 2019

Copyright (C) 2019 by author(s) and Scientific Research Publishing Inc. This work is licensed under the Creative Commons Attribution International License (CC BY 4.0). http://creativecommons.org/licenses/by/4.0/ c) (7) Open Access mals (C.Re.Di.Ma) performed the first inter-laboratory ring trial with the aim to standardize a diagnostic biomolecular approach for DMV in Italy. Viral isolation is usually considered the "gold standard" for the definitive diagnosis of most pathogens, but it is not often feasible in DMV diagnosis, due to the poor preservation of virus-targeted tissues in stranded cetacean carcasses, as well as to the lack of appropriate sensitivity of cell lines towards DMV variability. Therefore direct viral detection on tissues by means of reverse transcription-PCR (RT-PCR) represents a valuable option for DMV infection's diagnosis. For detecting DMV in cetacean die-offs occurred in the Mediterranean basin since 2013, C.Re.Di.Ma developed an RT-PCR based method targeting to a $287 \mathrm{bp}$ fragment of DMV nucleoprotein $(\mathrm{N})$ gene. With the purpose to evaluate its performances in terms of accuracy ( $\mathrm{Se}=$ sensitivity and $\mathrm{Sp}=$ specificity) and precision (reproducibility), it was submitted to a ring trial. So, 12 Public Laboratories belonging to the Italian dead stranded marine mammals diagnostic network were asked to analyze a panel of 40 samples (positive and negative for DMV, using different dilutions of a viral suspension obtained from a cell culture supernatant of a DMV strain) with the aforementioned technique. Furthermore, we also aimed at comparing the accuracy of other 7 molecular methods routinely applied for DMV detection in Italy. For this purpose, the second panel of identical $40 \mathrm{DMV}+\mathrm{ve}$ and -ve samples was provided to Laboratories that routinely used DMV detection methods other than those developed by C.Re.Di.Ma, in order to be analyzed simultaneously with the method they usually applied. The C.Re.Di.Ma technique showed high accuracy [mean $\mathrm{Se}=97.8 \%$ (95\% CI 84.2\% - 99.3\%), mean $\mathrm{Sp}=98.1 \%(95 \% \mathrm{CI} 72.5 \%-99.9 \%)]$ and very good precision $[\mathrm{k}$ combined equal to 0.91 (95\% CI 0.87 - 0.95)]. In conclusion, this study highlighted a satisfactory reliability of most of the molecular methods used in Italy for DMV detection.

\section{Keywords}

Dolphin Morbillivirus, RT-PCR, Interlab Ring Test, Method Comparison, Stranded Cetaceans, Mediterranean Sea

\section{Introduction}

Dolphin Morbillivirus (DMV), a single negative stranded RNA virus within the genus Morbillivirus, subfamily Paramyxovirinae, family Paramyxoviridae [1], is included in the cluster of Cetacean Morbillivirus (CeMV) [2]. DMV infection, in a similar manner to many other Morbillivirus genus members, affects mainly the upper respiratory tract as well as the central nervous system and the immune system of marine mammals [3] [4], having been associated with high mortality rates and stranding of cetaceans in different regions of the world [5]. In the Mediterranean Sea, two well documented DMV outbreaks occurred between 1990 and 1992 [6] as well as between 2006 and 2008 [7]. Moreover, DMV was deemed as the most likely cause of three cetacean unusual mortality events (UMEs) oc- 
curred since 2011 along Spanish [8] and Italian coastlines [9] [10].

Viral isolation and subsequent PCR identification are usually considered the "gold standard" for the definitive diagnosis of morbilliviral infections [4]. Nevertheless, this is often a challenging issue given the poor preservation of virus-targeted tissues when dealing with stranded cetacean carcasses; therefore, direct detection in tissues by means of reverse transcription-PCR (RT-PCR) followed by sequencing represents the first rapid, sensitive and specific tool for DMV detection. In the last few years, many conventional and Real-time RT-PCR methods have been developed to detect the presence of DMV worldwide [11] [12] [13].

In Italy, the National Reference Centre for diagnostic activities on dead stranded marine mammals (C.Re.Di.Ma) coordinates post mortem investigations on stranded cetaceans along the entire Italian peninsula, promoting the application of standardized guidelines regarding sampling and diagnostic techniques and the sharing of diagnostic results. In regard to direct DMV detection in tissues, C.Re.Di.Ma developed an RT-PCR restriction fragment length polymorphism (RFLP) technique, based upon the use of an RT-PCR with degenerate primers targeting a $287 \mathrm{bp}$ fragment of the nucleoprotein $(\mathrm{N})$ gene, followed by MseI RFLP analysis [14]. This work is aimed at assessing the performances of different diagnostic methods routinely applied in Italy for DMV infection's diagnosis. In this respect, identical panels of ad hoc samples were analyzed by the whole Italian dead stranded marine mammals' diagnostic network. More in detail, the accuracy ( $\mathrm{Se}=$ sensitivity and $\mathrm{Sp}=$ specificity) and precision (reproducibility) of the method described by Verna et al. 2017 [14] were verified and, at the same time, they were also compared with the accuracy of other 7 biomolecular techniques routinely applied for DMV detection in Italy [11] [12] [15]-[20].

\section{Material and Methods}

Twelve Public Diagnostic Laboratories belonging to the Italian diagnostic network on stranded cetaceans, listed in Table 1, took part in the ring trial. They constantly remained anonymous, thereby respecting privacy requirements and were named as L1 to L12. All the participating Laboratories were asked to apply the method proposed by C.Re.Di.Ma [14] on the first panel of samples. That method [14] was developed to detect a 287 bp sequence of a highly conserved region of Canine Distemper Virus (CDV) nucleoprotein (NP). Considering the relevant polymorphism of the target region, primers developed by Frisk et al.. 1999 [16] were redesigned through an in silico analysis. C.Re.Di.Ma, as the ring trial organizer, and in order to ensure the most consistent homogeneity of results, provided all of the participating Laboratories with detailed instructions and reagents to carry out the protocol described by Verna et al. More in detail, for total RNA extraction the Laboratories used the QIAamp Viral RNA Mini Kit (Qiagen) according to the manufacturer's instructions and reverse transcription 
Table 1. Laboratories involved in the ring trial listed in alphabetical order.

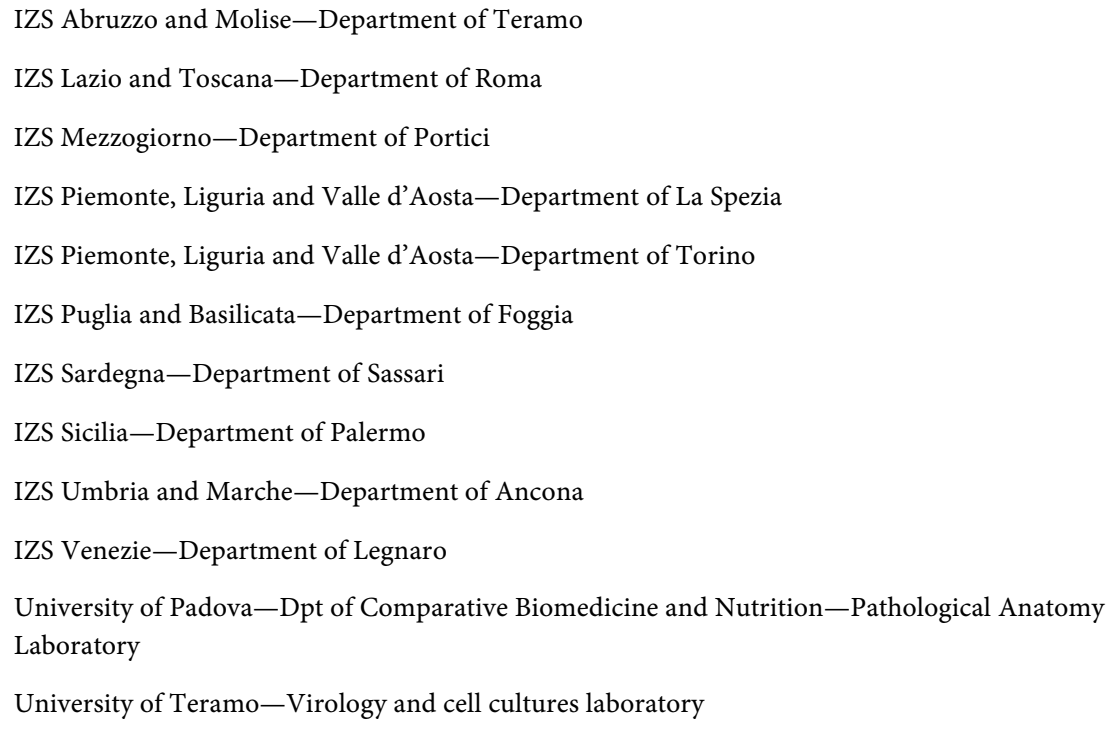

with simultaneous cDNA amplification was performed using the QIAGEN OneStep RT-PCR Kit (Qiagen). Eight Laboratories that routinely utilized a different method also examined a second panel of samples using their own protocols [11] [12] [15]-[20] whose essential details are reported in Table 2.

\subsection{Samples Preparation}

Each laboratory was provided with a panel composed of 40 samples to be analyzed with the method proposed by C.Re.Di.Ma (Panel 1: Samples from 1 to 40). Each panel consisted of 27 positive samples and 13 negative ones. C.Re.Di.Ma expected that its own method was able to reach values of $\mathrm{Se} \geq 80 \%$ and $\mathrm{Sp} \geq$ $90 \%$, therefore the number of positive and negative samples included in the study had to be sufficient to ensure that performance, with a limit lower than the $95 \%$ confidence interval for both indices was set at over $60 \%$. Laboratories that routinely employed other detection methods were also provided with a second panel containing the same number of samples, with distinguishable header and numbering (Panel 2: Samples from 51 to 90). The positive samples consisted in viral suspensions at different concentrations obtained from a cell culture infected with a given DMV isolate [21], recognized as the "gold standard". (GenBank Acc. No. MF589987). The virus was propagated on Vero/dogSLAM cell line [21] and after 6 days, when the cytopathic effect (CPE) affected $80 \%-90 \%$ of the cells, supernatants were collected and clarified by centrifugation at $3500 \mathrm{rpm}$. The initial concentration of the DMV isolate was determined on Vero/dogSLAM cell line with preformed monolayer and the final count of 103.5 TCID50/25 $\mu \mathrm{L}$ was calculated with the Reed-Muench method. Positive samples were set up at different viral concentrations (level 1 $=1: 20$ of the original supernatant, level $2=1$ : 100 and level $3=1: 500$ ). As negative control, a non-inoculated Vero/dogSLAM cell line supernatant was used under the same conditions. Samples were distributed 
Table 2. Method used by laboratories participating in the DMV ring trial.

\begin{tabular}{|c|c|c|c|}
\hline Methods & Target amplicon & primers & Laboratory \\
\hline $\begin{array}{l}\text { RT-PCR (Verna et al. 2017) } \\
\text { [14] }\end{array}$ & Gene N-287 bp & $\begin{array}{l}\text { MvF 5'-ACAGGATTGCKGAGGACCTAT-3' } \\
\text { MvR 5'-VARGATAACCATGTACGGTGC-3' }\end{array}$ & $\begin{array}{l}1,2,3,4,5,6,7,8 \\
\quad 9,10,11,12\end{array}$ \\
\hline Semi Nested RT-PCR [19] & Gene $\mathrm{P}-300 \mathrm{bp}$ & $\begin{array}{c}\text { MVP2202 5'-KKCTCRTGGTWCCWRCAGGC-3' } \\
\text { MVP2480R 5'-TCTCTYCTGTGCCCTTTTTAATGG-3' } \\
\text { internal } \\
\text { MVP A 5'-AGATGAGAGCTCTCTCGAGA-3' }\end{array}$ & 1 \\
\hline $\begin{array}{l}\text { Real-time RT-PCR TaqMan } \\
{[17][18]}\end{array}$ & Gene F-192 bp & $\begin{array}{l}\text { DMV Fu-F 5'-GGCACCATAATTAGCCAGGA-3' } \\
\text { DMV Fu-R 5'-GCCCAGATTTGTGCCTACAT-3' } \\
\text { probe unpublished }\end{array}$ & 2 \\
\hline RT-PCR [12] & Gene N-173 bp & $\begin{array}{l}\text { DMV-N-FP 5'-TGCCAGTACTCCAGGGAACATCCTTC-3' } \\
\text { DMV-N-RP 5'-TTGGGTCGTCAGTGTTGTCGGACCGTT-3' }\end{array}$ & 3,8 \\
\hline Real-time RT-PCR [20] & Gene $\mathrm{F}-45 \mathrm{bp}$ & $\begin{array}{c}\text { DMV-F3 5'-TCGCCGCAGACAAATGC-3' } \\
\text { DMV-R1 5'-TCCCTGGATCCCACTTGG-3' } \\
\text { probe } \\
\text { DMV-P2 -FAM-5'-CTTTAGTTGAGGTAGACGGAAT-MGBNFQ }\end{array}$ & 4 \\
\hline RT-PCR [15] & Gene $\mathrm{P}-429 \mathrm{bp}$ & $\begin{array}{l}\text { DMV-C 5'-ATGTTTATGATCACAGCGGT-3' } \\
\text { DMV-P2 5'-ATTGGGTTGCACCACTTGTC-3' }\end{array}$ & 5 \\
\hline $\begin{array}{l}\text { Real time RT-PCR sybrgreen } \\
\qquad[16]\end{array}$ & Gene $N-287$ bp & $\begin{array}{l}\text { P-1 (CDV) 5'-ACAGGATTGCTGAGGACCTAT-3' } \\
\text { P-2 (CDV) 5'-CAAGATAACCATGTACGGTGC-3' }\end{array}$ & 9 \\
\hline Nested PCR [11] & $\begin{array}{c}\text { Gene } \mathrm{H}-612 \\
\text { bp-nested } 200 \mathrm{bp}\end{array}$ & $\begin{array}{l}\text { DMV-11F 5'-CCGAACCTGATGATCCATTT-3' } \\
\text { DMV-11R 5'-CGTAAATGTCCATCCCTGCT-3' } \\
\text { internal } \\
\text { DMV-13F 5'-CATCATAGGGGGTGGTTTGA-3' } \\
\text { DMV-13R 5'-GGGGTGGTCTACTCTTGCAC-3' }\end{array}$ & 11 \\
\hline
\end{tabular}

in aliquots of $250 \mu \mathrm{l}$, identified individually with numerical code and stored at $-80^{\circ} \mathrm{C}$

\subsection{Homogeneity and Stability Tests on Ring Trial Samples}

Homogeneity in sample preparation was evaluated by analyzing with the method Verna et al. 2017 [14] five replicates for each level of positivity under the same conditions planned for the execution of ring trial analyses (Figure 1). Extractions were carried out using the QIAamp viral RNA mini kit QIAGEN and retrotranscription and amplification kit OneStep RT-PCR Qiagen. Evaluation of homogeneity was performed at the end of the preparation of all samples and prior to their shipment to all laboratories involved in the ring trial. Five aliquots for each virus concentration were randomly selected from every group of samples ready for distribution. In order to eliminate possible confounding factors, tests were carried out under strict standard conditions; therefore they were performed by the same operator and in the same analytical session. Acceptability criteria for homogeneity tests were set up at reproduction, on each selected aliquot, of the same amplicon yield, corresponding to their respective reference samples for each viral dilution, including negative ones. With reference to stability tests, aliquots for each level of virus concentration were used to perform two different trials. In order to simulate the effect of potential temperature failure 


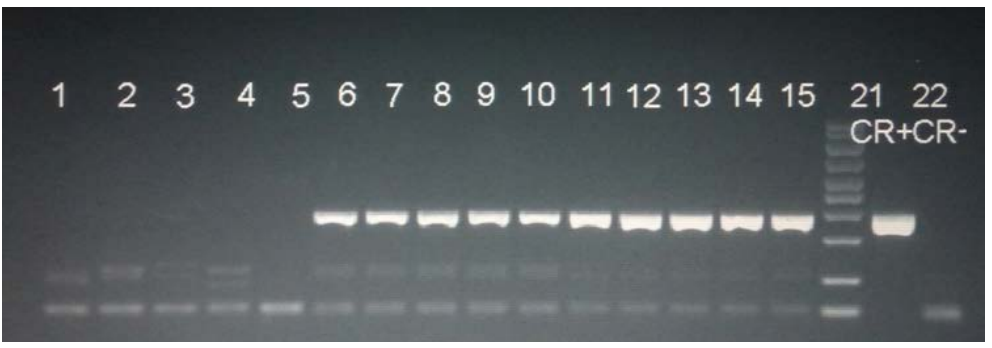

(a)

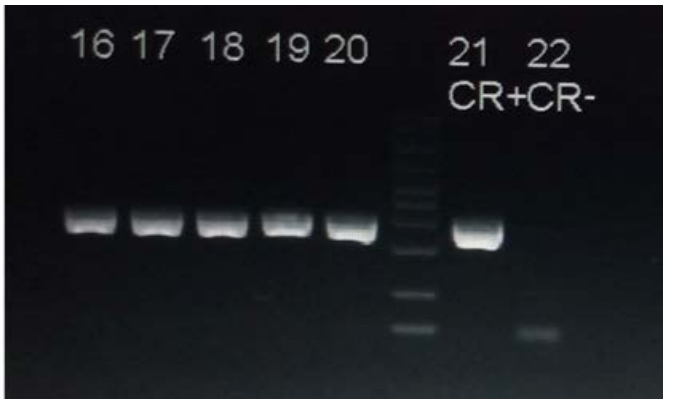

(b)

Figure 1. Homogeneity test.

occurrences during shipment on virus samples, a stability test was designed to evaluate the resistance of samples to stress temperature for each level of positivity, including the negative ones and it was carried out before the shipment. Analyses were performed on three replicates for each virus concentration maintained at room temperature at: $t_{0}=$ corresponding to homogeneity test day; $t_{1}=$ day1; $t_{2}=$ day $2 ; t_{3}=$ day3 (Figure 2 ). A second stability test was carried out to verify the sample stability at the storage conditions $\left(-80^{\circ} \mathrm{C}\right)$, during the entire period of execution of the ring trial. In this case, three replicates for every single virus concentration (including negative controls) were analyzed, every 15 days, from the day of shipment until the defined closing time for results submission. Acceptability criteria for stability tests were also set up at reproduction, during all ring-testing period under all storage and stress conditions, on each selected aliquot, of the same amplicon yield, corresponding to their respective reference samples for each viral dilution, including negative ones.

\subsection{Statistical Analysis}

Performances of each laboratory in the application of the method described by Verna et al. were evaluated in terms of both accuracy and precision (reproducibility). Conversely, for each of the other 7 methods routinely applied throughout the Country, accuracy was evaluated. In order to assess the accuracy, Sensitivity (Se) and Specificity (Sp), positive (PPV) and negative (NPV) predictive values were estimated, as well as their 95\% confidence intervals (95\% CI). To assess precision Cohen's kappa was pairwise estimated for each participant towards everyone, as well as for each participant with respect to the majority judgment (MJ, i.e. the outcome given by most of participants for each sample examined). 


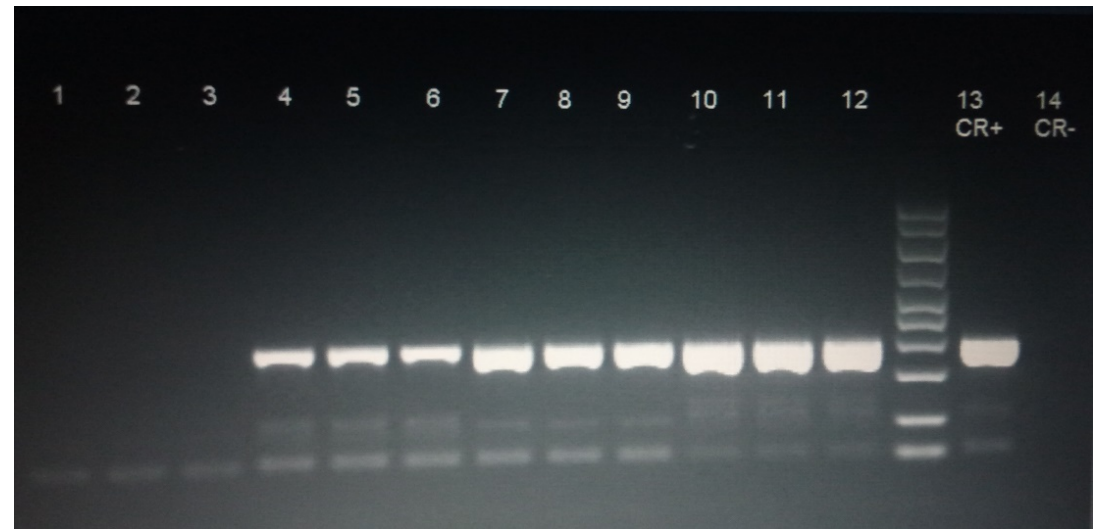

Figure 2. Stability test under stress temperature conditions.

Moreover, the k-combined was calculated [22] to evaluate the agreement between all the participants in the trial. Statistical analysis was carried out using the software STATA 15 software (StataCorp, College Station, Texas, USA).

\section{Results}

\subsection{Samples Homogeneity and Stability}

Outcomes satisfied the acceptability requirements, thereby confirming expectations, as summarized in Figure 1 and Figure 2.

All dilution samples gave back the expected results. Pictures show: in Figure 1(a) five replicates of negative samples (lanes 1 to 5); five replicates of DMV at 1:500 dilution (lanes 6 to 10); five replicates of DMV at 1:100 dilution (lanes 11 to 15); in Figure 1(b) five replicates of DMV at dilution 1:20 (lanes 16 to 20) (Amplicon size: 287 bp, Agarose gel: 2\%, DNA ladder: AmpliSize Molecular Ruler 50 bp - 2000 bp BioRad, USA)

Replicates of each viral dilution, kept at room temperature, were examined in triplicates at the onset $\left(\mathrm{t}_{0}\right)$ and at $24\left(\mathrm{t}_{1}\right), 48\left(\mathrm{t}_{2}\right)$ and $72\left(\mathrm{t}_{3}\right)$ hours from the shipment. Presence of viral RNA was always confirmed in all replicates containing DMV with the appropriate yield at each time interval. Figure 2 only shows amplicons obtained from each DMV dilution, as well as negative replicates, extracted and amplified after storage at room temperature for 72 hours $\left(t_{3}\right)$ : Negative samples (lanes 1 to 3); DMV at 1:500 dilution (lanes 4 to 6); DMV at 1:100 dilution (lanes 7 to 9); DMV at dilution 1:20 (lanes 10 to 12). (Amplicon size: 287 bp, Agarose gel: 2\%, DNA ladder: AmpliSize Molecular Ruler 50 bp - 2000 bp BioRad, USA)

For stability test at storage conditions, replicates of each viral dilution, stored at $-80^{\circ} \mathrm{C}$, were examined after 0,15 and 30 days to cover all testing period until the deadline for results' submission. Presence of viral RNA was confirmed in all replicates containing DMV at each time interval.

\subsection{PCR Results}

This study allowed us to verify the performances of most of molecular detection 
methods applied within the Italian dead stranded marine mammals' diagnostic network. In particular, the technique by Verna et al. 2017, proposed by C.Re.Di.Ma, showed high accuracy values; sensitivity, specificity and predictive values obtained by each participating Laboratory are shown in Figure 3 and Figure 4 . The average accuracy values assessed on the Verna et al. method resulted as $\mathrm{Se}=97.8 \%(95 \%$ CI $84.2 \%-99.3 \%), \mathrm{Sp}=98.1 \%(95 \%$ CI $72.5 \%-$ 99.9\%), PPV = 99\% (95\% CI 85.4\% - 100\%), NPV = 96.6\% (71.4\% - 99\%).

With reference to the other methods, the Nested PCR method (Gene H-612 bp - $200 \mathrm{bp}$ ) showed difficulties both in identifying positive samples ( $\mathrm{Se}=66.7 \%$; $\mathrm{CI}=46 \%-83.5 \%)$ and negative ones $(\mathrm{Sp}=76.9 \%$; $\mathrm{CI}=46.2 \%-95 \%)$, although there was not enough significative statistical evidence to infer that the performances were lower than the method by Verna et al. For the rtRT-PCR SYBR green method only it was possible to state that the ability to correctly identify negative samples $(\mathrm{Sp}=15.4 \%$; $\mathrm{CI}=1.9 \%-45.4 \%)$ was significantly lower than the other methods. The estimates of Cohen's kappa calculated either for each pair of participants or for each participant versus the majority judgment, using the Verna et al. technique, are shown in supplementary material (Table S1, Table S2). The precision of the results obtained by all the Laboratories involved in the herein reported ring trial using the method by Verna et al. 2017 ( $\mathrm{k}$ combined) was equal to 0.91 ( $95 \%$ CI $0.87-0.95)$.

\section{Discussion}

As previously stated, DMV represents one of the most relevant threats to free-ranging cetaceans. Different RT-PCR assays have been used for DMV and,
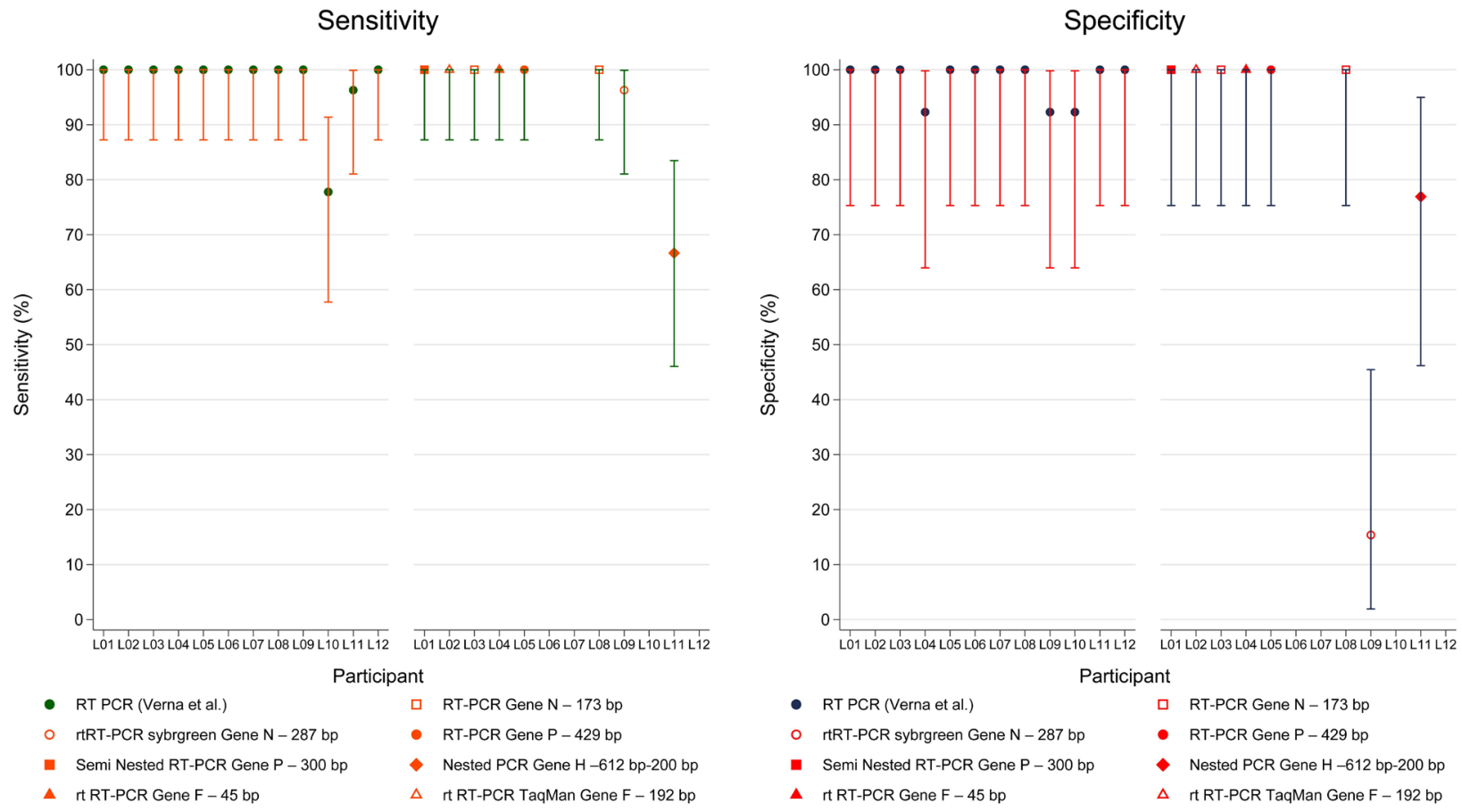

Figure 3. Sensitivity and specificity and their 95\% confidence intervals (95\% CI). 

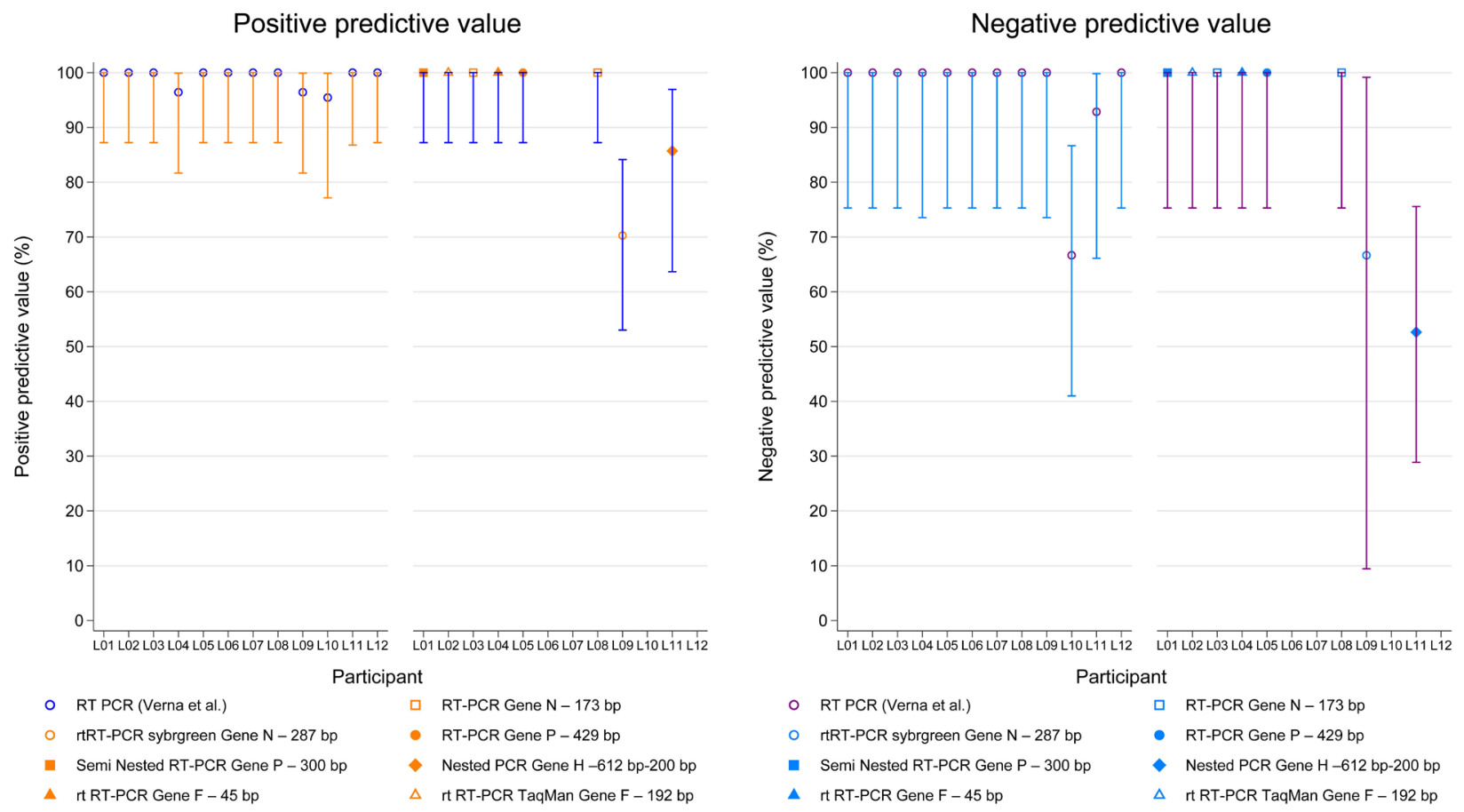

Figure 4. Positive and negative predictive values and their $95 \%$ confidence intervals (95\% CI).

more in general, for CeMV detection from stranded cetaceans worldwide. Within such context, the consistent advances in molecular biology have allowed a progressively faster, easier and more reliable development of biomolecular protocols for DMV infection's laboratory diagnosis [4]. The main goal of the herein reported interlaboratory ring trial was to assess the diagnostic performances of molecular assays to detect DMV used by 12 different Public Laboratories belonging to the Italian diagnostic stranding network. To the best of our knowledge, this is the first study performed to evaluate the accuracy of different biomolecular methods for DMV detection worldwide. The aforementioned network has been recently involved in the management of 2 cetacean UMEs [9] [10] respectively caused by the Mediterranean and the North-Eastern Atlantic DMV strains. The method described by Verna et al. 2017, applied by C.Re.Di.Ma routinely as well as during both the aforementioned UMEs, turned out to be a reliable and relatively inexpensive diagnostic tool, easily applicable and suitable to characterize different morbilliviral genotypes, as amplicons are appropriate to be submitted to RFLP analysis by mean of MseI Enzyme. First of all, our study was aimed at evaluating the accuracy and reproducibility of such technique. The method was successfully applied by all the participating Laboratories and high accuracy values were obtained. Even though two Labs (L10-L11) faced sensitivity-related issues and three others (L4-L9-L10) specificity-related ones, there was no statistically significant evidence to infer that the performances among the participants were different. In fact, the concordance values obtained by the various participants were fully satisfactory, provided that the lower limit of the confidence interval was at levels considered by the literature from "almost perfect" 
to "excellent" [22]. The accuracy values of the other 7 methods tested were satisfactory too. Most laboratories obtained high values. We additionally noted that the Laboratory that applied the Nested PCR method (Gene H-612 bp - 200 bp) showed difficulties both in identifying positive samples ( $\mathrm{Se}=66.7 \%$; $\mathrm{CI}=46 \%$ $83.5 \%)$ and negative ones ( $\mathrm{Sp}=76.9 \%$; $\mathrm{CI}=46.2 \%$ - 95\%), but, even in this case, there was not enough statistical evidence to infer that the concerned Laboratory had lower performances than with the method by Verna et al. 2017. Only for the Real-time RT-PCR (rtRT-PCR) SYBR green (Gene N-287 bp) method it was possible to verify that the ability to correctly identify the negative samples ( $\mathrm{Sp}=$ $15.4 \%$; CI $=1.9 \%-45.4 \%$ ) was lower than the other methods. This might have resulted from cross-reaction or cross contamination, which are potential hazards in routine laboratory diagnostics, as also reported in other ring tests. Comparison of the sensitivities of different methods showed that the probability that the positive result given by the rtRT-PCR SYBR green (Gene N-287 bp) method was correct was 71\% (CI = 52\% - 84\%), whereas for the method by Verna et al. 2017 it was at least $95 \%(\mathrm{CI}=85 \%-100 \%)$. With reference to the negative results that were generally obtained by means of the rtRT-PCR SYBR green method (Gene $\mathrm{N}-287 \mathrm{bp}$ ), they also had low reliability: the probability that a negative sample was correctly classified could vary from $10 \%$ to $100 \%$. The experimental conditions under which every test was carried out showed good reliability of the diagnostic methods. Notwithstanding the above, the results of laboratory analyses under field conditions could be affected by other factors, such as the genetic variability within between the different morbillivirus genus members and CeMV/DMV strains, along with the poor preservation status of tissues frequently found on stranded animals. Within such context, the stability test allowed us to highlight the remarkable resistance of morbilliviral agents under both correct and stressed preservation conditions. In this respect, the good stability of viral genomes, observed under unsuitable storage conditions, leads us to suppose that the "morbillivirus-related samples" preservation degree may not be "dramatically" affected during transfer between different laboratories for confirmatory analyses and comparison of results.

\section{Conclusion}

Based upon the results of the herein described ring trial, we can conclude that most of the biomolecular techniques used in our Country for DMV infection's diagnosis show a satisfactory reliability and reproducibility, while an adequate level of expertise appears to be also present in all of the participating Laboratories regarding the application of the method by Verna et al. 2017, which could be recommended as a reliable RT-PCR protocol to be used for the routine laboratory diagnosis of DMV infection in Italy.

\section{Acknowledgements}

This study was funded by the Italian Ministry of Health within the framework of 
the Research Project "IZS PLV 12/15 RC". We would like to warmly thanks all the other Referents for Marine Mammal Strandings of Istituti Zooprofilattici Sperimentali (Terracciano G., Cocumelli C., Scholl F., Di Nocera F., Lucifora G. Puleio R., Pintore A., Petrella A., Di Francesco G., Ferri N., Latini M., Gavaudan S., Toffan A., Rubini S.) for their efforts in the standardization of the diagnostic approach on stranded cetaceans.

\section{Conflicts of Interest}

The authors declare no conflicts of interest regarding the publication of this paper.

\section{References}

[1] ICTV (2015) Virus Taxonomy: 2014 Release.

[2] Jo, W.K., Kruppa, J., Habierski, A., Van De Bildt, M., Mazzariol, S., Di Guardo, G., Siebert, U., Kuiken, T., Jung, K., Osterhaus, A. and Ludlow M. (2018) Evolutionary Evidence for Multi-Host Transmission of Cetacean Morbillivirus. Emerging Microbes and Infections, 7, 1-15. https://doi.org/10.1038/s41426-018-0207-x

[3] Beineke, A., Siebert, U., Wohlsein, P. and Baumgärtner, W. (2010) Immunology of Whales and Dolphins. Veterinary Immunology and Immunopathology, 133, 81-94. https://doi.org/10.1016/j.vetimm.2009.06.019

[4] Van Bressem, M.F., Duignan, P., Banyard, A., Barbieri, M., Colegrove, K., De Guise, S., Di Guardo, G., Dobson, A., Domingo, M., Fauquier, D., Fernandez, A., Goldstein, T., Grenfell, B., Groch, K., Gulland, F., Jensen, B., Jepson, P., Hall, A., Kuiken, T., Mazzariol, S., Morris, S., Nielsen, O., Raga, J., Rowles, T., Saliki, J., Sierra, E., Stephens, N., Stone, B., Tomo, I., Wang, J., et al. (2014) Cetacean Morbillivirus: Current Knowledge and Future Directions. Viruses, 6, 5145-5181. https://doi.org/10.3390/v6125145

[5] Van Bressem, M.F., Van Waerebeek, K. and Raga, J.A. (1999) A Review of Virus Infections of Cetaceans and the Potential Impact of Morbilliviruses, Poxviruses and Papillomaviruses on Host Population Dynamics. Diseases of Aquatic Organisms, 38, 53-65. https://doi.org/10.3354/dao038053

[6] Aguilar, A. and Raga, J.A. (1993) The Striped Dolphin Epizootic in the Mediterranean Sea. Ambio, 22, 524-528

[7] Raga, J.A., Banyard, A., Domingo, M., Corteyn, M., Van Bressem, M.F., Fernández, M., Aznar, F.J. and Barrett, T. (2008) Dolphin Morbillivirus Epizootic Resurgence, Mediterranean Sea. Emerging Infectious Diseases, 14, 471-473. https://doi.org/10.3201/eid1403.071230

[8] Rubio-Guerri, C., Melero, M., Esperón, F., Bellière, E.N., Arbelo, M., Crespo, J.L., Sierra, E., García-Párraga, D. and Sánchez-Vizcaíno, J.M. (2013) Unusual Striped Dolphin Mass Mortality Episode Related to Cetacean Morbillivirus in the Spanish Mediterranean Sea. BMC Veterinary Research, 9, Article ID: 6903. https://doi.org/10.1186/1746-6148-9-106

[9] Casalone, C., Mazzariol, S., Pautasso, A., Di Guardo, G., Di Nocera, F., Lucifora, G., Ligios, C., Franco, A., Fichi, G., Cocumelli, C., Cersini, A., Guercio, A., Puleio, R., Goria, M., Podestà, M., Marsili, L., Pavan, G., Pintore, A., De Carlo, E., Eleni, C. and Caracappa, S. (2014) Cetacean Strandings in Italy: An Unusual Mortality Event along the Tyrrhenian Sea Coast in 2013. Diseases of Aquatic Organisms, 109, 81-86. https://doi.org/10.3354/dao02726

[10] Pautasso, A., Iulini, B., Grattarola, C., Giorda, F., Goria, M., Peletto, S., Masoero, L., 
Mignone, W., Varello, K., Peterella, A., Carbone, A., Pintore, A., Denurra, D., Sholl, F., Cersini, A., Puleio, R., Purpari, G., Lucifora, G., Fusco, G., Di Guardo, G., Mazzariol, S. and Casalone, C. (2019) Novel Dolphin Morbillivirus (DMV) Outbreak among Mediterranean Striped Dolphins Stenella coeruleoalba in Italian Waters. Diseases of Aquatic Organisms, 132, 215-220.

https://doi.org/10.3354/dao03323

[11] Centelleghe, C., Beffagna, G., Zanetti, R., Zappulli, V., Di Guardo, G. and Mazzariol, S. (2016) Molecular Analysis of Dolphin Morbillivirus: A New Sensitive Detection Method Based on Nested RT-PCR. Journal of Virological Methods, 235, 85-91. https://doi.org/10.1016/j.jviromet.2016.05.005

[12] Grant, R.J., Banyard, A.C., Barrett, T., Saliki, J.T. and Romero, C.H. (2009) Real-Time RT-PCR Assays for the Rapid and Differential Detection of Dolphin and Porpoise Morbilliviruses. Journal of Virological Methods, 156, 117-123. https://doi.org/10.1016/j.jviromet.2008.11.008

[13] Sierra, E., Zucca, D., Arbelo, M., García-Álvarez, N., Andrada, M., Déniz, S. and Fernández, A. (2014) Fatal Systemic Morbillivirus Infection in Bottlenose Dolphin, Canary Islands, Spain. Emerging Infectious Diseases Journal, 20, 269-271.

[14] Verna, F., Giorda, F., Miceli, I., Rizzo, G., Pautasso, A., Romano, A., Iulini, B., Pintore, M.D., Mignone, W., Grattarola, C., Bozzetta, E., Varello, K., Dondo, A., Casalone, C. and Goria, M. (2017) Detection of Morbillivirus Infection by RT-PCR RFLP Analysis in Cetaceans and Carnivores. Journal of Virological Methods, 247, 22-27. https://doi.org/10.1016/j.jviromet.2017.05.009

[15] Barrett, T., Visser, I.K., Mamaev, L., Goatley, L., Van Bressem, M.F. and Osterhaust, A.D. (1993) Dolphin and Porpoise Morbilliviruses Are Genetically Distinct from Phocine Distemper Virus. Virology, 193, 1010-1012. https://doi.org/10.1006/viro.1993.1217

[16] Frisk, A.L., König, M., Moritz, A. and Baumgärtner, W. (1999) Detection of Canine Distemper Virus Nucleoprotein RNA by Reverse-Transcription-PCR Using Serum, Whole Blood, and Cerebrospinal Fluid from Dogs with Distemper. Journal of Clinical Microbiology, 37, 3634-3643.

[17] Rubio-Guerri, C., Esperón, F., Melero, M., Bellière, E.N., Arbelo, M.. Crespo, J.L., Sierra, E., García-Parraga, D. and Sánchez-Vizcaíno, J.M. (2012) An Unusual Mortality Episode in the Spanish Mediterranean Sea Related to Cetacean Morbillivirus (CeMV).40 th Symposium of the European Association for Aquatic Mammals, Madrid, España, 9-12 August 2012.

[18] Bellière, E.N., Esperón, F., Arbelo, M., Muñoz, M.J., Fernández, A. and Sánchez-Vizcaíno, J.M. (2010) Presence of Herpesvirus in Striped Dolphins Stranded during the Cetacean Morbillivirus Epizootic along the Mediterranean Spanish Coast in 2007. Archives of Virology, 155, 1307-1311.

https://doi.org/10.1007/s00705-010-0697-x

[19] Sierra, E., Sánchez, S., Saliki, J.T., et al. (2014) Retrospective Study of Etiologic Agents Associated with Nonsuppurative Meningoencephalitis in Stranded Cetaceans in the Canary Islands. Journal of Clinical Microbiology, 52, 2390-2397. https://doi.org/10.1128/JCM.02906-13

[20] Viscardi, M., Cozzolino, L., Brandi, S., Marati, L., Valentino, M.P., Lucifora, G., Balestrieri, A. and Fusco, G. (2017) Preliminary Phylogenetic Analysis of a Cetacean Morbillivirus Causing Outbreak in Calabria, Southern Italy. 18 th Conference of the World Association of Veterinary Laboratory Diagnosticians (WAVLD), Sorrento, Italy, 7-10 June 2017, 123.

[21] Peletto, S., Caruso, C., Cerutti, F., Modesto, P., Biolatti, C., Pautasso, A., Grattarola, 
C., Giorda, F., Mazzariol, S., Mignone, W., Masoero, L., Casalone, C. and Acutis, P.L. (2018) Efficient Isolation on Vero.DogSLAMtag Cells and Full Genome Characterization of Dolphin Morbillivirus (DMV) by Next Generation Sequencing. Scientific Reports, 8, Article No. 860. https://doi.org/10.1038/s41598-018-19269-2

[22] Fleiss, J.L., Levin, L. and Paik, M.C. (2003) Statistical Methods for Rates and Proportions. 3rd Edition, John Wiley \& Sons, New York, USA.

https://doi.org/10.1002/0471445428 


\section{Supplementary Materials}

Table S1. Cohen's k estimated for each pair of participants using the method Verna et al.

\begin{tabular}{|c|c|c|c|c|}
\hline \multirow[b]{2}{*}{ Lab A } & \multirow[b]{2}{*}{ Lab B } & \multicolumn{3}{|c|}{ confidence interval $95 \%$} \\
\hline & & k Coen & lower limit & upper limit \\
\hline \multirow[t]{11}{*}{ L01 } & L02 & 1.00 & 0.69 & 1.00 \\
\hline & L03 & 1.00 & 0.69 & 1.00 \\
\hline & LO4 & 0.94 & 0.63 & 1.00 \\
\hline & L05 & 1.00 & 0.69 & 1.00 \\
\hline & L06 & 1.00 & 0.69 & 1.00 \\
\hline & L07 & 1.00 & 0.69 & 1.00 \\
\hline & L08 & 1.00 & 0.69 & 1.00 \\
\hline & Lo9 & 0.94 & 0.63 & 1.00 \\
\hline & L10 & 0.64 & 0.34 & 0.94 \\
\hline & L11 & 0.94 & 0.63 & 1.00 \\
\hline & $\mathrm{L} 12$ & 1.00 & 0.69 & 1.00 \\
\hline \multirow[t]{10}{*}{ L02 } & L03 & 1.00 & 0.69 & 1.00 \\
\hline & LO4 & 0.94 & 0.63 & 1.00 \\
\hline & L05 & 1.00 & 0.69 & 1.00 \\
\hline & Lo6 & 1.00 & 0.69 & 1.00 \\
\hline & L07 & 1.00 & 0.69 & 1.00 \\
\hline & L08 & 1.00 & 0.69 & 1.00 \\
\hline & LO9 & 0.94 & 0.63 & 1.00 \\
\hline & L10 & 0.64 & 0.34 & 0.94 \\
\hline & L11 & 0.94 & 0.63 & 1.00 \\
\hline & $\mathrm{L} 12$ & 1.00 & 0.69 & 1.00 \\
\hline \multirow[t]{9}{*}{ L03 } & L04 & 0.94 & 0.63 & 1.00 \\
\hline & L05 & 1.00 & 0.69 & 1.00 \\
\hline & L06 & 1.00 & 0.69 & 1.00 \\
\hline & L07 & 1.00 & 0.69 & 1.00 \\
\hline & L08 & 1.00 & 0.69 & 1.00 \\
\hline & Lo9 & 0.94 & 0.63 & 1.00 \\
\hline & L10 & 0.64 & 0.34 & 0.94 \\
\hline & L11 & 0.94 & 0.63 & 1.00 \\
\hline & $\mathrm{L} 12$ & 1.00 & 0.69 & 1.00 \\
\hline \multirow[t]{8}{*}{ LO4 } & L05 & 0.94 & 0.63 & 1.00 \\
\hline & LO6 & 0.94 & 0.63 & 1.00 \\
\hline & L07 & 0.94 & 0.63 & 1.00 \\
\hline & L08 & 0.94 & 0.63 & 1.00 \\
\hline & LO9 & 0.88 & 0.57 & 1.00 \\
\hline & L10 & 0.58 & 0.29 & 0.88 \\
\hline & L11 & 0.89 & 0.58 & 1.00 \\
\hline & $\mathrm{L} 12$ & 0.94 & 0.63 & 1.00 \\
\hline \multirow[t]{7}{*}{ L05 } & LO6 & 1.00 & 0.69 & 1.00 \\
\hline & L07 & 1.00 & 0.69 & 1.00 \\
\hline & L08 & 1.00 & 0.69 & 1.00 \\
\hline & LO9 & 0.94 & 0.63 & 1.00 \\
\hline & L10 & 0.64 & 0.34 & 0.94 \\
\hline & L11 & 0.94 & 0.63 & 1.00 \\
\hline & L12 & 1.00 & 0.69 & 1.00 \\
\hline \multirow[t]{6}{*}{ L06 } & LO7 & 1.00 & 0.69 & 1.00 \\
\hline & L08 & 1.00 & 0.69 & 1.00 \\
\hline & LO9 & 0.94 & 0.63 & 1.00 \\
\hline & L10 & 0.64 & 0.34 & 0.94 \\
\hline & L11 & 0.94 & 0.63 & 1.00 \\
\hline & L12 & 1.00 & 0.69 & 1.00 \\
\hline \multirow[t]{5}{*}{ L07 } & L08 & 1.00 & 0.69 & 1.00 \\
\hline & LO9 & 0.94 & 0.63 & 1.00 \\
\hline & L10 & 0.64 & 0.34 & 0.94 \\
\hline & L11 & 0.94 & 0.63 & 1.00 \\
\hline & $\mathrm{L} 12$ & 1.00 & 0.69 & 1.00 \\
\hline \multirow{4}{*}{ L08 } & LO9 & 0.94 & 0.63 & 1.00 \\
\hline & L10 & 0.64 & 0.34 & 0.94 \\
\hline & L11 & 0.94 & 0.63 & 1.00 \\
\hline & L12 & 1.00 & 0.69 & 1.00 \\
\hline \multirow[t]{3}{*}{ LO9 } & L10 & 0.58 & 0.29 & 0.88 \\
\hline & L11 & 0.89 & 0.58 & 1.00 \\
\hline & $\mathrm{L} 12$ & 0.94 & 0.63 & 1.00 \\
\hline \multirow[t]{2}{*}{ L10 } & L11 & 0.69 & 0.39 & 0.99 \\
\hline & $\mathrm{L} 12$ & 0.64 & 0.34 & 0.94 \\
\hline L11 & L12 & 0.94 & 0.63 & 1.00 \\
\hline
\end{tabular}


Table S2. Cohen's k against the majority judgment (MJ) for each participant using the method Verna et al.

\begin{tabular}{|c|c|c|c|}
\hline & & \multicolumn{2}{|c|}{ confidence interval 95\% } \\
\hline Laboratory & Cohen's kappa & lower limit & upper limit \\
\hline L01 & 1.00 & 0.69 & 1.00 \\
\hline L02 & 1.00 & 0.69 & 1.00 \\
\hline L01 & 1.00 & 0.69 & 1.00 \\
\hline L02 & 1.00 & 0.69 & 1.00 \\
\hline L03 & 1.00 & 0.69 & 1.00 \\
\hline L04 & 0.94 & 0.63 & 1.00 \\
\hline L05 & 1.00 & 0.69 & 1.00 \\
\hline L06 & 1.00 & 0.69 & 1.00 \\
\hline L07 & 1.00 & 0.69 & 1.00 \\
\hline L08 & 1.00 & 0.69 & 1.00 \\
\hline L09 & 0.94 & 0.63 & 1.00 \\
\hline L10 & 0.64 & 0.34 & 0.94 \\
\hline L11 & 0.94 & 0.63 & 1.00 \\
\hline L12 & 1.00 & 0.69 & 1.00 \\
\hline
\end{tabular}

